This document is the Accepted Manuscript version of a Published Work that appeared in final form in Biochemistry, ( 2016 American Chemical Society after peer review and technical editing by publisher. To access the final edited and published work see Huang, P. J. J., Vazin, M., \& Liu, J. (2016). In Vitro Selection of a DNAzyme Cooperatively Binding Two Lanthanide lons for RNA Cleavage. Biochemistry, 55(17), 2518-2525. https://doi.org/10.1021/acs.biochem.6b00132

\title{
In Vitro Selection of a DNAzyme Cooperatively Binding Two Lanthanide lons for RNA Cleavage
}

\author{
Po-Jung Jimmy Huang ${ }^{1}$, Mahsa Vazin ${ }^{1}$, and Juewen Liu* \\ ${ }^{1}$ Equal contribution
}
Department of Chemistry, Waterloo Institute for Nanotechnology
University of Waterloo

200 University Ave West, Waterloo, Ontario, Canada, N2L 3G1

Fax: (+1) 519-746-0435

E-mail: liujw@uwaterloo.ca

\section{Funding}

Funding for this work was from the Natural Sciences and Engineering Research Council (NSERC) of Canada (386326 and STPGP 447472-13). 


\begin{abstract}
Trivalent lanthanide ions $\left(\mathrm{Ln}^{3+}\right)$ were recently employed to select RNA-cleaving DNAzymes, and three new DNAzymes have been reported so far. In this work, dysprosium $\left(\mathrm{Dy}^{3+}\right)$ was used with a library containing 50 random nucleotides. After six rounds of in vitro selection, a new DNAzyme named Dy10a was obtained and characterized. Dy10a has a bulged hairpin structure cleaving a RNA/DNA chimeric substrate. Dy10a is highly active in the presence of the five $\mathrm{Ln}^{3+}$ ions in the middle of the lanthanide series $\left(\mathrm{Sm}^{3+}, \mathrm{Eu}^{3+}, \mathrm{Gd}^{3+}, \mathrm{Tb}^{3+}\right.$ and $\left.\mathrm{Dy}^{3+}\right)$, while its activity descends on the two sides. The cleavage rate reaches $0.6 \mathrm{~min}^{-1}$ at $\mathrm{pH} 6$ with just $200 \mathrm{nM} \mathrm{Sm}^{3+}$, which is the fastest among all known $\mathrm{Ln}^{3+}$-dependent enzymes. Dy10a binds two $\mathrm{Ln}^{3+}$ ions cooperatively. When a phosphorothioate (PS) modification is introduced at the cleavage junction, the activity is dropped by more than 2500 -fold for both the $R_{\mathrm{p}}$ and $S_{\mathrm{p}}$ diastereomers; and thiophilic $\mathrm{Cd}^{2+}$ cannot rescue the activity. The $\mathrm{pH}$-rate profile has a slope of 0.37 between $\mathrm{pH} 4.2$ to 5.2, and the slope was even less at higher $\mathrm{pH}$. Based on these data, a model of metal binding is proposed. Finally, a catalytic beacon sensor is constructed which can detect $\mathrm{Ho}^{3+}$ down to 1.7 nM.
\end{abstract}


Since $1994,{ }^{1}$ in vitro selection has been used to isolate RNA-cleaving DNAzymes. DNAzymes are attractive because of their excellent catalytic efficiency, stability, cost-effectiveness, and programmability. $^{2-5}$ Divalent metal ions were conceived to be an indispensable part of DNAzyme catalysis. ${ }^{2,6,7}$ The first DNAzyme was isolated in the presence of $\mathrm{Pb}^{2+}$ for $\mathrm{RNA}$ cleavage. ${ }^{1}$ Many subsequent selections were carried out with physiological metals $\left(\mathrm{Mg}^{2+}\right.$ and $\mathrm{Ca}^{2+}$ ) for intracellular RNA cleavage. ${ }^{8-11}$ To facilitate spectroscopic studies and further promote cleavage, transition metal ions were also employed, such as $\mathrm{Mn}^{2+}, \mathrm{Co}^{2+}, \mathrm{Zn}^{2+}$ and $\mathrm{Cu}^{2+},{ }^{2,12-16}$ For environmental monitoring, selections were also carried out with toxic heavy metals such as $\mathrm{Ag}^{+}$, $\mathrm{Cd}^{2+}, \mathrm{Hg}^{2+}$ and $\mathrm{UO}_{2}{ }^{2+} \cdot{ }^{17-20}$

In contrast, trivalent metals have not been systematically explored until recently. We hypothesize that trivalent lanthanide ions $\left(\mathrm{Ln}^{3+}\right)$ might be efficient for RNA cleavage, since $\mathrm{Ln}^{3+}$ are hard Lewis acids with strong affinity to DNA phosphate. In fact, even free $\mathrm{Ln}^{3+}$ can catalyze RNA cleavage, although it requires a high metal concentration with little sequence specificity. ${ }^{21}$ The effect of $\mathrm{Ln}^{3+}$ was studied in a few known enzymes. For example, $\mathrm{Ln}^{3+}$ enhances the activity of the leadzyme by several folds. ${ }^{22}$ The GR5 DNAzyme can also be moderately activated by $\mathrm{Ln}^{3+} .^{23}$ On the other hand, $\mathrm{Ln}^{3+}$ inhibits the hammerhead ribozyme, ${ }^{24}$ and the 8-17 DNAzyme. ${ }^{25}$ In addition to RNA cleavage, $\mathrm{Ln}^{3+}$ ions also catalyze other reactions such as DNA cleavage and RNA ligation. ${ }^{26,27}$

Given these examples, we reasoned that in vitro selection in the presence of $\mathrm{Ln}^{3+}$ might produce new enzymes. Recently, we reported four $\mathrm{Ln}^{3+}$-dependent RNA-cleaving DNAzymes. ${ }^{28-}$ ${ }^{31}$ The Ce13d DNAzyme was isolated using a $\mathrm{Ce}^{4+}$ salt and an $\mathrm{N}_{50}$ DNA library (e.g., a library with 50 random nucleotides). ${ }^{28} \mathrm{Ce} 13 \mathrm{~d}$ has a similar activity across the whole $\mathrm{Ln}^{3+}$ series (but is inactive with $\mathrm{Ce}^{4+}$ ); and it also requires $\mathrm{Na}^{+} \cdot{ }^{32-34} \mathrm{Next}$, Lu12 was isolated using a $\mathrm{N}_{35}$ library in 
the presence of $\mathrm{Lu}^{3+}$, a heavy lanthanide. Interestingly, Lu12 is more active with the light $\mathrm{Ln}^{3+}$, showing a descending activity trend with the last few heavy $\operatorname{Ln}^{3+}{ }^{29}$ This pattern is similar to another DNAzyme cleaving $2^{\prime}-5^{\prime}$ linked RNA. ${ }^{31}$ Finally, using the $\mathrm{N}_{35}$ library, we carried out three selections in the presence of $\mathrm{Ho}^{3+}, \mathrm{Er}^{3+}$ and $\mathrm{Tm}^{3+}$, respectively. A new DNAzyme named $\operatorname{Tm} 7$ was isolated. $\mathrm{Tm} 7$ is nearly inactive with the first seven light $\mathrm{Ln}^{3+}$ but is highly active with the heavy ones. ${ }^{30} \mathrm{Tm} 7$ binds three $\mathrm{Ln}^{3+}$ ions cooperatively, which has never been observed previously for DNAzymes.

To date, our $\mathrm{N}_{50}$ library was used only once with a light lanthanide $(\mathrm{Ce})$. In our continuous effort to search for new $\mathrm{Ln}^{3+}$-dependent DNAzymes, we herein employed the $\mathrm{N}_{50}$ library with a heavy lanthanide, dysprosium $\left(\mathrm{Dy}^{3+}\right)$. Dysprosium strongly absorbs neutrons and it has a high magnetic susceptibility, allowing applications in nuclear reactors and data storage. A new DNAzyme, named Dy10, was isolated and characterized. It has very high affinity for $\mathrm{Ln}^{3+}$, binding two $\mathrm{Ln}^{3+}$ cooperatively for catalysis.

\section{Materials and Methods}

Chemicals. The in vitro selection related DNA and the beacon DNA samples were from Integrated DNA Technologies (Coralville, IA). All the other DNAs were from Eurofins (Huntsville, AL). See Table S1 and S2 for DNA sequence details. The lanthanides and other metal salts were from Sigma-Aldrich at the highest available purity. Tris(hydroxymethyl)aminomethane (Tris), 2-(N-morpholino)ethanesulfonic acid (MES), 2-[4-(2hydroxyethyl)piperazin-1-yl]ethanesulfonic acid (HEPES), 3-(N-morpholino)propansulfonic acid (MOPS), EDTA disodium salt dihydrate, sodium chloride, sodium acetate, and ammonium 
acetate were from Mandel Scientific Inc. (Guelph, Ontario, Canada). In vitro selection related molecular biology reagents were from New England Biolabs.

In vitro selection. The methods for in vitro selection, PCR, cloning, and sequencing were previously reported, ${ }^{28}$ and are not repeated here. The only difference was that $\mathrm{Dy}^{3+}$ was used to induce cleavage. The selection conditions for each round are presented in Table S3. The round 6 library was cloned and sequenced, yielding a total of 40 sequences.

Gel-based assays. DNAzyme activity assays were performed with the FAM-labeled substrate (Sub-FAM, $0.7 \mu \mathrm{M}$ ) and the Dy10a enzyme (or other enzyme sequences, $1.1 \mu \mathrm{M}$ ). The DNAzyme complex was annealed in buffer A (50 mM MES, pH 6.0, $25 \mathrm{mM} \mathrm{NaCl}$ ) before adding a metal solution to initiate the cleavage reaction. For the $\mathrm{pH}$-dependent studies, the acetate, MES and MOPS buffers $(50 \mathrm{mM}$ with $25 \mathrm{mM} \mathrm{NaCl})$ were used. The reaction products were separated on a denaturing polyacrylamide gel (dPAGE) and analyzed using a Bio-Rad ChemiDoc MP imaging system. Separation of the two diastereomers of the PS-modified substrate was performed following our previously reported procedure. ${ }^{17}$

Sensing. The sensor performance was studied using a microplate reader (SpectraMax M3). The sensor complex was formed by annealing the FAM-labeled substrate and the quencher-labeled enzyme (1:1.5 ratio) in buffer A. In each well, $100 \mu \mathrm{L}$ of the complex containing $50 \mathrm{nM}$ FAMlabeled substrate was diluted in $10 \mathrm{mM}$ HEPES (pH 7.5). $1 \mu \mathrm{L}$ of metal ion was added after 5 min of background reading and the signaling kinetics was monitored. 


\section{Results and Discussion}

In vitro DNAzyme selection. With a DNA library containing 50 random nucleotides $\left(\mathrm{N}_{50}\right)$, we carried out in vitro selection in the presence Dy ${ }^{3+}$ following a previously established method. ${ }^{28}$ The whole library had only a single RNA linkage serving as the putative cleavage site, while the rest are DNA. At each round, a fraction of the library may undergo self-cleavage after adding $\mathrm{Dy}^{3+}$. This cleaved population may contain catalytically active sequences and they were harvested using gel electrophoresis and amplified by PCR. The incubation time and $\mathrm{Dy}^{3+}$ concentration for each round are shown in Table S3. After six rounds of selection, 67\% cleavage was achieved (Figure 1A).

Secondary structure analysis. The round 6 library was cloned and sequenced, yielding 40 sequences. The majority of the library (85\%) are similar to the previously reported Ce13d and Lu12 DNAzymes (see Table S4 for DNA sequence alignment). ${ }^{28,29}$ This is not surprising, since both Ce13d and Lu12 are quite active with $\mathrm{Dy}^{3+}$. For the remaining four sequences, two are identical (Dy1 and Dy17) but inactive (Figure S1). The other two, Dy10 and Dy35, are very similar (Figure 1E). Figure 1B shows the secondary structure of Dy10 predicted by Mfold, ${ }^{35}$ and the cleavage site is marked with the arrowhead. This cis-cleaving structure can be easily converted to the trans-cleaving form by removing the nucleotides in the gray box. We also replaced the nucleotides in the red box by a smaller six-nucleotide loop, generating a truncated DNAzyme named Dy10a (see Figure 1C).

In addition to the hairpin, Dy10a has two loops. The smaller loop is an $A A G G$ tetranucleotide. The other sequence (Dy35) has GAGG in this part (underlined in Figure 1E). For the larger loop, Dy10 and Dy35 differ only by one nucleotide as well. Therefore, these two highly conserved loops might be important for catalysis. To monitor cleavage, the substrate 
strand in Figure 1C was labeled with a FAM (carboxyfluorescein). Indeed, Dy10a is active (Figure 1D, lane 1). We also converted Dy35 into a trans-cleaving form and it is also active (the last lane in Figure 1D). Since Dy10a appears to be different from all the previously reported $\mathrm{Ln}^{3+}$-dependent DNAzymes, ${ }^{28-30}$ we characterized it in detail.
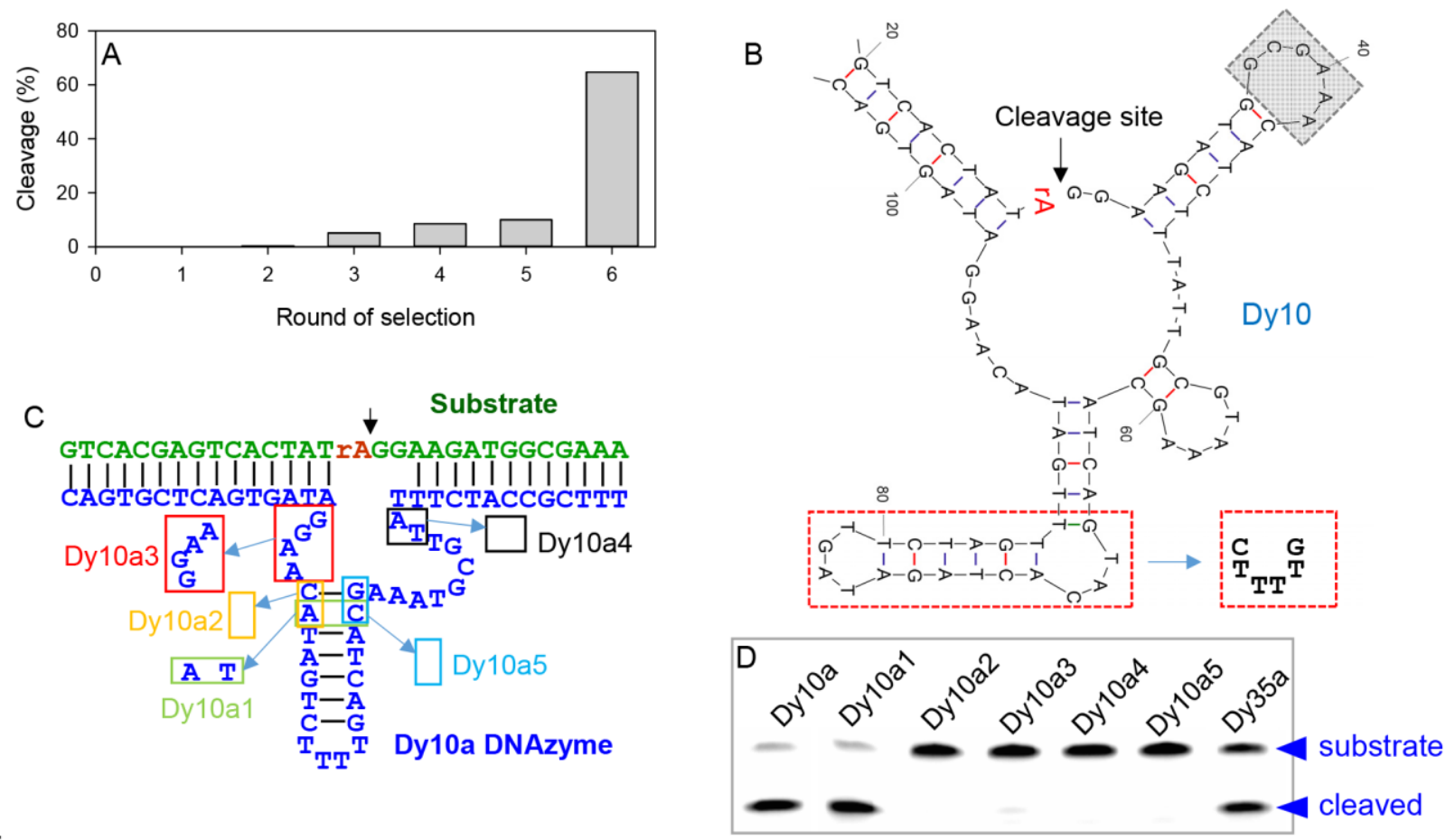

E

Dy10 rAGGAAGATGGCGAAACATCTTTATTGCGTAAAGCATCAGTACACTAGATAGTTCTAGTTTGATACAAGGATAGT Dy35 rAGGAAGATGGCGAAACATCTT-ATTGCGTAAGCCGAAATGGGCGTTAGAGTACGAAACATTCAGCGAGGTTAGT

Figure 1. (A) Progress of the $\mathrm{Dy}^{3+}$-dependent selection. (B) The secondary structure of the ciscleaving Dy10 predicted by Mfold. For a trans-cleaving DNAzyme, the nucleotides in the shaded box are removed and the nucleotides in the red box are replaced by the six nucleotides next to it. (C) The trans-cleaving Dy10a. Various mutations/truncations are also shown, where the boxed nucleotides are replaced. (D) A gel image showing activity of the mutants after $1 \mathrm{~h}$ reaction with $0.5 \mu \mathrm{M} \mathrm{Sm}{ }^{3+}$. (E) Sequence alignment of Dy10 and Dy35 starting from the cleavage site rA. The nucleotides in the two loops are shown in boldface; the underlined nucleotides highlight the difference. 
With only two active sequences, the information from the DNA library is limited, only supporting the overall secondary structure and two mutable nucleotides. We performed rational mutation studies to test if further truncations are possible. In Dy10a, we assigned the $A-C$ mismatch to be part of the stem region in the hairpin (see Figure 1C, green box). To confirm this, we mutated the $C$ to $T$ (named Dy10a1) forming a Watson-Crick base pair, and it is still very active (Figure 1D). Therefore, this $A-C$ mismatch is indeed part of the hairpin. We next switched the $A A G G$ tetranucleotide to GGAA (Dy10a3), and the activity was lost (note that $G A G G$ is active). It appears that these purines cannot be randomly changed. Next, we gradually truncated a few nucleotides (Dy10a2, 4, 5), and they all inhibited the activity. Therefore, Dy10a appears to be an optimal construct already and further truncations cannot be made.

Dy10a is highly specific for $\mathbf{L n}^{3+}$. A criterion to confirm a new DNAzyme is to study the $\mathrm{Ln}^{3+}-$ dependent activity trend. For example, the Lu12 DNAzyme has descending activity for the last few heavy $\mathrm{Ln}^{3+}$, while the $\mathrm{Tm} 7$ is active only with the seven heavy $\mathrm{Ln}^{3+}$. For this purpose, the Dy10a DNAzyme was incubated for 5 min with the $14 \mathrm{Ln}^{3+}$ ions $(1 \mu \mathrm{M}$ each). All the samples induced cleavage (Figure 2A), while the most active ones are in the middle of the series (e.g., from $\mathrm{Sm}^{3+}$ to $\mathrm{Dy}^{3+}$ ). Next, we quantified the cleavage at $1 \mathrm{~min}$ and 5 min (Figure 2B). A bellshaped activity pattern is obtained, which is different from all the previous DNAzymes. In addition, Dy10a is also quite efficient, cleaving $>30 \%$ substrate in $1 \mathrm{~min}$ at $\mathrm{pH} 6\left(1 \mu \mathrm{M} \mathrm{Sm}^{3+}\right)$.

Next, the activity of Dy10a with 21 other metal ions was studied. With $5 \mu \mathrm{M}$ metal (see Figure $2 \mathrm{C}$ ), only $\mathrm{Y}^{3+}$ showed activity. $\mathrm{Y}^{3+}$ has a size similar to $\mathrm{Ho}^{3+}$ and it can activate the previously reported DNAzymes. ${ }^{28-30}$ Interestingly, $5 \mu \mathrm{M} \mathrm{Pb}^{2+}$ did not show any cleavage. Based on previous studies, $\mathrm{Pb}^{2+}$ is active with many DNAzymes. ${ }^{1,8,28,36}$ With $100 \mu \mathrm{M}$ metal (see Figure 
$3 \mathrm{~B}$ ), the activity of $\mathrm{Y}^{3+}$ dropped significantly due to inhibition, while $\mathrm{Pb}^{2+}$ showed moderate cleavage. Overall, Dy10a is highly specific for $\mathrm{Ln}^{3+}$.

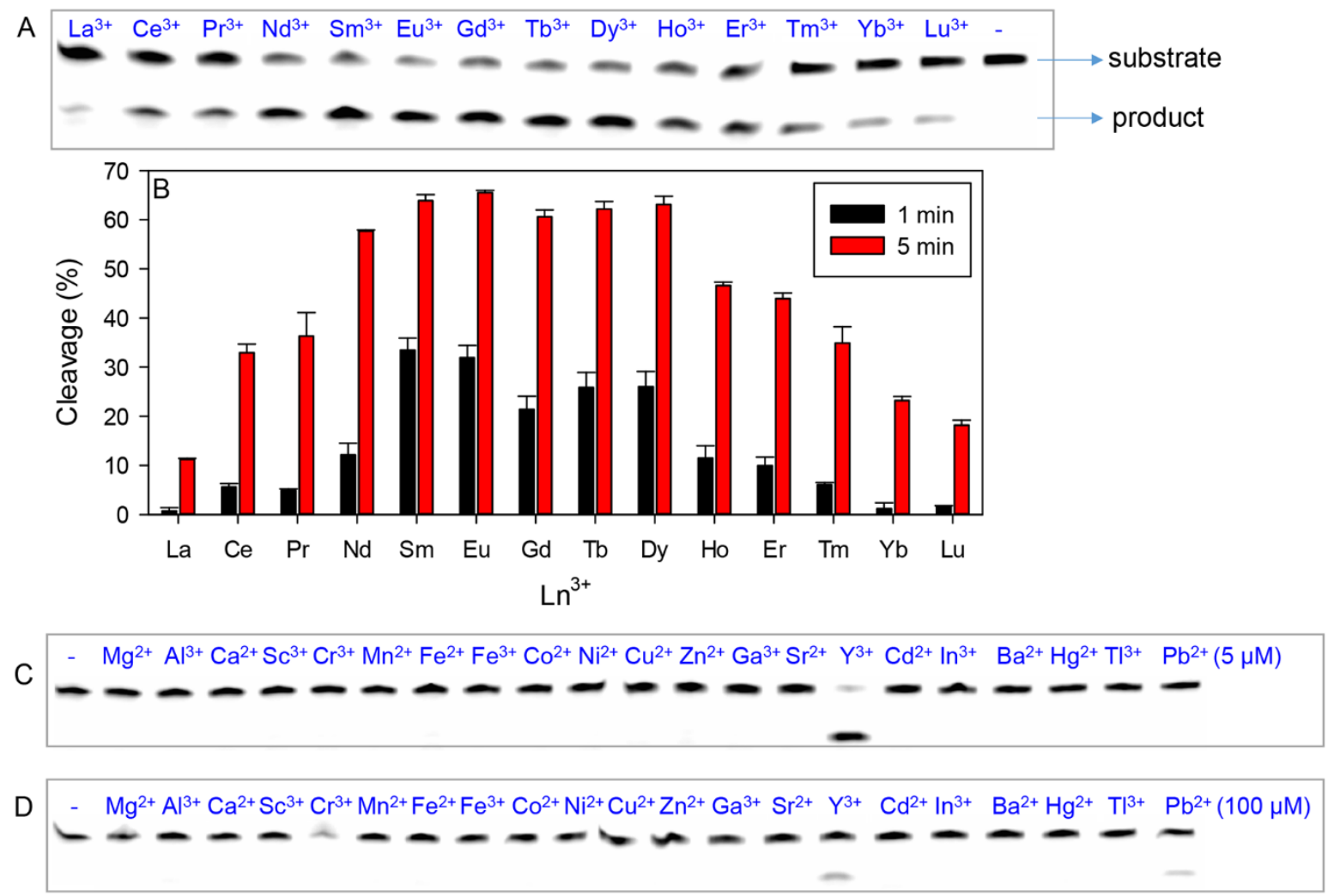

Figure 2. (A) A gel image of Dy10a cleavage in the presence of $1 \mu \mathrm{M} \mathrm{Ln}^{3+}$ for $5 \mathrm{~min}$ at $\mathrm{pH} 6$. The last lane is the negative control without $\mathrm{Ln}^{3+}$. (B) The percentage of substrate cleavage in the presence of $1 \mu \mathrm{M} \mathrm{Ln}{ }^{3+}$ after 1 and 5 min reaction. Gel images of Dy10a metal specificity test in the presence of (C) $5 \mu \mathrm{M}$ and (D) $100 \mu \mathrm{M}$ of various metal ions at $\mathrm{pH} 6.0$ for $1 \mathrm{~h}$.

Dy10a has strong affinity for $\mathbf{L n}^{3+}$. After confirming metal specificity, a lanthanide concentration-dependent study was carried out. $\mathrm{Sm}^{3+}$ was chosen for this study since it is one of the most active metals for Dy10a (Figure 2B). A few kinetic traces are shown in Figure 3A; each was fitted to a first order kinetics. The rate constants are plotted in Figure 3B, which displays a 
sigmoidal trend suggesting metal binding cooperativity. We then plotted the logarithm of rate against the logarithm of $\mathrm{Sm}^{3+}$ concentration (see Figure 3C). The highest activity occurs between 0.5 and $1 \mu \mathrm{M} \mathrm{Sm}^{3+}$. At higher concentrations, $\mathrm{Sm}^{3+}$ starts to inhibit the activity. The apparent dissociation constant $\left(K_{\mathrm{d}}\right)$ from this gel-based assay is $0.1 \mu \mathrm{M} \mathrm{Sm}^{3+}$, making it one of the tightest metal binding DNAzymes. The initial linear part was fitted to a straight line with a slope of 1.8, suggesting two metal ions operating cooperatively for catalysis.

Most previously reported RNA-cleaving DNAzymes or ribozymes bind only a single metal ion when probed by enzyme activity. ${ }^{69}$ Dinuclear $\mathrm{Ln}^{3+}$ species for RNA cleavage was previously reported (slope $=\sim 2$ ) using their free metal salts (no DNAzymes) ${ }^{21,37}$ However, those rates were very slow even with a high concentration of $\mathrm{Ln}^{3+}$ (e.g., a few $\mathrm{mM}$ ). Cooperativity, using mixtures of $\mathrm{Zn}^{2+}$ and $\mathrm{Sn}^{4+}$, was reported but the rate was extremely slow $\left(\sim 0.003 \mathrm{~min}^{-1}\right){ }^{38}$ Some large ribozymes use multiple metal ions but without cooperativity. ${ }^{39-41}$ It was proposed that the VS ribozyme binds four $\mathrm{Mg}^{2+}$ ions, but the affinity is very low $\left(K_{\mathrm{d}}=17 \mathrm{mM}\right) \cdot{ }^{42} \mathrm{We}$ recently reported that the Tm7 DNAzyme employs three $\mathrm{Ln}^{3+}$ ions cooperatively. ${ }^{30}$ In this study, Dy10a employs two $\mathrm{Ln}^{3+}$ ions, further supporting the assertion that it is a new DNAzyme. Dy10a is also a very efficient enzyme; its rate reaches $\sim 0.7 \mathrm{~min}^{-1}$ with $0.5 \mu \mathrm{M} \mathrm{Sm}{ }^{3+}$, the highest among all reported $\mathrm{Ln}^{3+}$-dependent DNAzymes at $\mathrm{pH} 6$.

This Dy10 sequence only appeared twice in the final 40 sequences we obtained. Compared to Ce13d or Lu12 (85\% of the final library), Dy10 has much higher activity at low metal concentrations. For example, with $0.5 \mu \mathrm{M}$ metal, Dy10 is $>10$ times faster than the other two DNAzymes. The reason that Dy10 failed to dominate the library is attributed to its more easily inhibited by $\mathrm{Ln}^{3+}$. For example, its activity is significantly suppressed with $10 \mu \mathrm{M} \mathrm{Dy}{ }^{3+}$ (see Figure S2). For comparison, Ce13d works optimally with $10 \mu \mathrm{M} \mathrm{Ln}^{3+} .{ }^{28}$ We used 10 to 50 
$\mu \mathrm{M} \mathrm{Dy}{ }^{3+}$ during this selection. Had the selection been carried out with a lower concentration of $\mathrm{Dy}^{3+}$, we speculate that more Dy10 sequences would have been present in the final library.
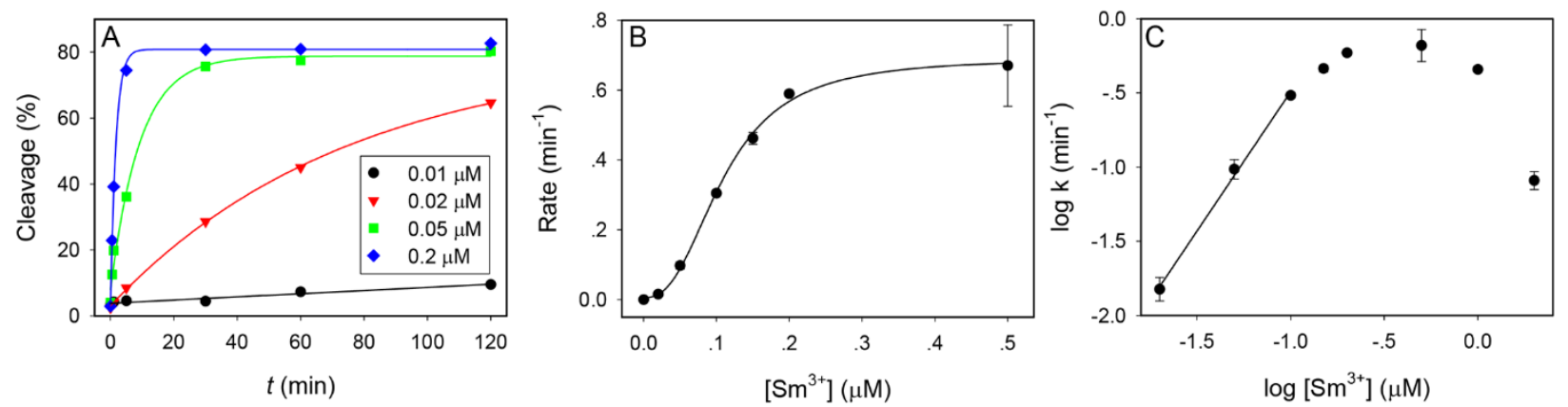

Figure 3. (A) The Dy10a DNAzyme cleavage kinetics at a few $\mathrm{Sm}^{3+}$ concentrations at $\mathrm{pH}$ 6. (B) Cleavage rate as a function of $\mathrm{Sm}^{3+}$ concentration. The trend is sigmoidal suggesting cooperative metal binding. (C) A double log plot with an initial slope of 1.8, suggesting two metal binding. At higher metal concentrations, the rate drops due to DNAzyme inhibition by $\mathrm{Sm}^{3+}$.

pH-dependent activity. To further characterize Dy10a, a pH-dependent study was performed. Figure 4 plots the log of cleavage rate as a function of $\mathrm{pH}$. Overall, the rate increases with increasing $\mathrm{pH}$, and we divided the whole $\mathrm{pH}$ range into three distinct segments. From $\mathrm{pH} 4.2$ to $\mathrm{pH}$ 5.4, the slope is 0.37 . After that, the rate remained essentially constant (slope $=0.03$ ) until $\mathrm{pH}$ 7.2. Further increase of $\mathrm{pH}$ even decreased the rate. Most RNA-cleaving ribozymes and DNAzymes have a slope of one for the $\mathrm{pH}$-rate profile; this single deprotonation step is often attributed to activation of the $2^{\prime}-\mathrm{OH}$ nucleophile of the ribose. ${ }^{43}$ This is certainly not the case for Dy10a. Such bell-shaped pH-rate profile with the initial slope much less than 1 is reminiscent of the VS ribozyme. In the VS ribozyme, a guanine acts as a general base to assist deprotonation of the $2^{\prime}-\mathrm{OH} \cdot{ }^{44,45}$ At the same time, an adenine was proposed as a general acid to donate a proton to 
the oxygen on the leaving group. The $\mathrm{pH}$ needs to be high enough to deprotonate the guanine $\left(\mathrm{p} K_{\mathrm{a}}=9.2\right.$ normally) but still low enough to protonate the $\mathrm{N} 1$ position of the adenine $\left(\mathrm{p} K_{\mathrm{a}}=3.5\right.$ normally). The optimal catalysis is achieved when the product of the acidic and basic species is the highest, which explains the bell-shaped $\mathrm{pH}$-rate profile. The role of metal ions is to shift the $\mathrm{p} K_{\mathrm{a}}$ of these bases.

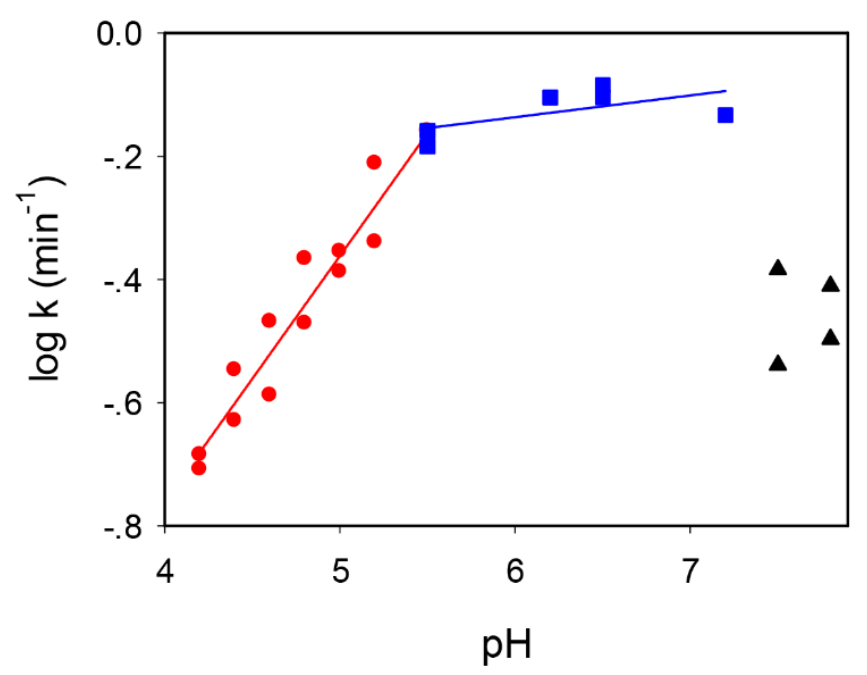

Figure 4. pH-dependent cleavage rate of the Dy10a DNAzyme in the presence of $0.5 \mu \mathrm{M} \mathrm{Sm}^{3+}$. The $\mathrm{pH}$ range was divided into three segments based on the slopes. The buffers used were 50 $\mathrm{mM}$ acetate ( $\mathrm{pH} 4.2$ to 5.0) MES ( $\mathrm{pH} 5.0$ to 6.5) and MOPS (pH 6.5 to 7.8) with $25 \mathrm{mM} \mathrm{NaCl.}$

We hypothesize that similar mechanisms might take place in Dy10a. The $\mathrm{p} K_{\mathrm{a}}$ value of water bound to $\mathrm{Sm}^{3+}$ is $8.61 .^{46}$ In the $\mathrm{pH}$ range we studied, this water should remain charge neutral and may serve as a general acid. At present, it is unclear whether the general acid and base roles are carried out by two nucleobases (there are a few guanines and adenines in the enzyme loops), or by $\mathrm{Ln}^{3+}$. To confirm the importance of the $2^{\prime}-\mathrm{OH}$ group, we also tested substrates with $2^{\prime}-\mathrm{F}$ (fluorine modified) and $2^{\prime}-\mathrm{OCH}_{3}$ at the ribose ring, and neither can be 
cleaved by Dy10a in the presence of $\mathrm{Sm}^{3+}$ (see Figure S3). Therefore, the reaction still relies on the 2 '-OH. To confirm the cleavage product, we carried out further mass spectrometry studies. The 5'-fragment of the substrate has a cyclic phosphate, which is the standard product from the 2'-OH nucleophilic attack (see Figure S4).

Using $2 \mathrm{mM} \mathrm{La}^{3+}$ at $\mathrm{pH} 8.7$ (no DNA), Chin and co-workers suggested a dimeric $\mathrm{La}^{3+}$ species that cleaves RNA at a rate of $\sim 0.6 \mathrm{~min}^{-1} .{ }^{37}$ The $\mathrm{pH}$-rate slope was 5 , which was explained by metal ion hydrolysis and formation of the dimeric species required for catalysis. In another report, Matsumura and Komiyama used $5 \mathrm{mM} \mathrm{Ln}^{3+}$. They also proposed a dinuclear $\mathrm{Nd}^{3+}$ complex, and the $\mathrm{pH}$-rate slope was $\sim 2$ from $\mathrm{pH} 7$ to $9 .^{21,47}$ Although the Dy10a DNAzyme also employs two $\mathrm{Sm}^{3+}$ ions, our results are quite different from those using free $\mathrm{Ln}^{3+}$ ions in a few aspects. First, our metal concentration was only $0.5 \mu \mathrm{M} \mathrm{Sm}^{3+}$, which was 4,000 to 10,000 times lower than the previous work using free $\mathrm{Ln}^{3+}$. Second, our studies were mainly carried out from $\mathrm{pH} 4.2$ to 7 instead of above $\mathrm{pH} 7$ by the others. Note that in our $\mathrm{pH}$ range, the free $\mathrm{Ln}^{3+}$ ions are essentially inactive for RNA cleavage. Due to the lower $\mathrm{pH}$ and $\mathrm{Ln}^{3+}$ concentration, it is less likely for $\mathrm{Sm}^{3+}$ to dimerize on its own to account for the two metal ion catalysis we observed. Instead, the DNAzyme scaffold must play a critical role to assist binding of the two metals. This may explain the difference in the $\mathrm{pH}$ trend. For free lanthanide ions, high $\mathrm{pH}$ and high metal concentration are required to form dimers.

Dy10a has a very large thio effect. Lanthanide ions are hard Lewis acids that like to bind to phosphate. Thus, phosphorothioate (PS) modification is a powerful method to probe such metal binding. ${ }^{48-50}$ We replaced one of the non-bridging oxygen atoms at the cleavage junction phosphate by sulfur. By doing so, two diastereoiosmers were produced (referred to as $R_{\mathrm{p}}$ and $S_{\mathrm{p}}$ in Figure 5A). We separated them by HPLC following a previously reported method. ${ }^{17}$ Most 
known RNA-cleaving enzymes bind metal using the pro- $R_{\mathrm{p}}$ oxygen; when this oxygen is replaced by sulfur, the activity is significantly suppressed (typically by $30-200$ fold). ${ }^{32,48-51}$ On the other hand, if the pro- $S_{\mathrm{p}}$ oxygen is replaced by sulfur, the enzyme remains quite active (with only a several fold drop in activity).

The Dy10a rate with the $R_{\mathrm{p}} \mathrm{PS}$ substrate is $0.013 \mathrm{~h}^{-1}$ (see Figure $5 \mathrm{~B}$, solid dots), which is $>2500$ times slower than the normal PO substrate. Intriguingly, the $R_{\mathrm{p}}$ rate is similarly slow (only approximately two-fold faster than the $S_{\mathrm{p}}$ rate). This is very different from most previously reported enzymes, where the $S_{\mathrm{p}}$ is typically faster by two orders of magnitude than the $R_{\mathrm{p}}$ using hard metal ions. This suggests that both non-bridging oxygen atoms might be used equally to bind metal, which is also consistent with Dy10a binding two metals. If both oxygen atoms are involved, replacing either one by sulfur will have a large effect on the rate.

$\mathrm{Cd}^{2+}$, a thiophilic metal, is commonly used rescue enzyme activity after the PS modification. When $\mathrm{Cd}^{2+}$ was used, the activity was still quite slow (Figure 5D). Interestingly, activity with the $S_{\mathrm{p}}$ form became slightly faster than with the $R_{\mathrm{p}}$ form. The fact that $\mathrm{Cd}^{2+}$ also works but at similarly slow rates further suggests that both non-bridging oxygen atoms are involved.

This non-typical PS behavior might be related to the binding of two metal ions for catalysis. For comparison, the Tm7 DNAzyme binds three lanthanide ions and it is completely inactive with the PS modification, regardless of the $R_{\mathrm{p}}$ or $S_{\mathrm{p}}$ form, and regardless of the type of metal ion tested. ${ }^{30}$ On the other hand, most DNAzymes/ribozymes binding one metal have partially retained activity with the PS substrate and the isomer can often be fully rescued by $\mathrm{Cd}^{2+}$. 

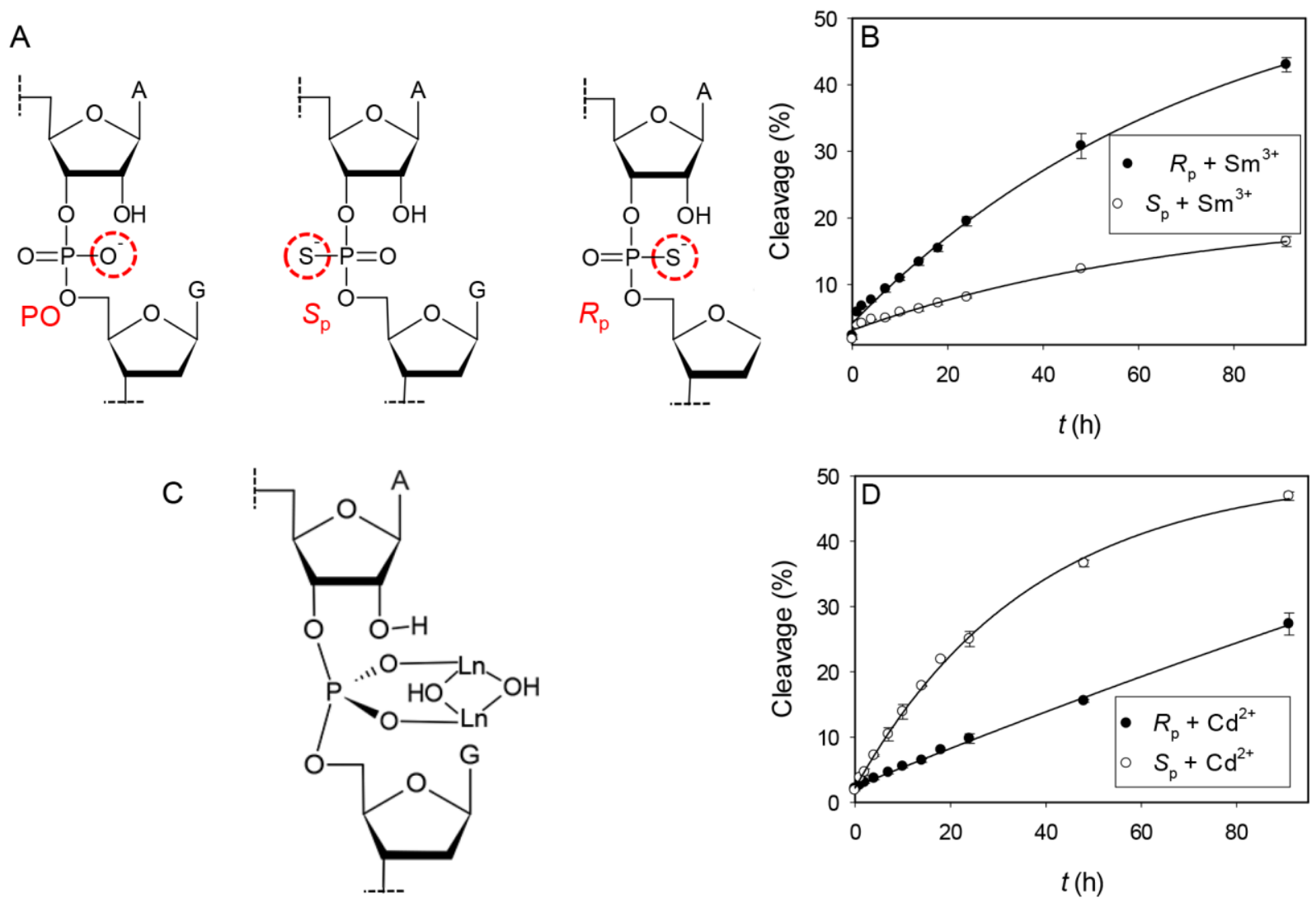

Figure 5. (A) A scheme of the normal PO, $R_{\mathrm{p}}$ and $S_{\mathrm{p}}$ cleavage junctions. Cleavage kinetics of the $R_{\mathrm{p}}$ and $S_{\mathrm{p}}$ PS substrate by (B) $0.5 \mu \mathrm{M} \mathrm{Sm}^{3+}$ or (D) $10 \mu \mathrm{M} \mathrm{Cd}^{2+}$ in $50 \mathrm{mM} \mathrm{MES}$ (pH 6.0) with 25 $\mathrm{mM} \mathrm{NaCl}$. Note these reactions were carried out up to 4 days. (C) A proposed scheme of a dinuclear lanthanide complex binding the cleavage site phosphate in Dy10a.

Based on the above biochemical studies, we proposed a metal binding scheme in Figure 5C. This scheme is inspired by the mechanism proposed by Komiyama and co-workers for free lanthanide ions. ${ }^{21}$ Two $\mathrm{Sm}^{3+}$ ions are involved in the catalysis, and the PS-substitution experiment suggests that each $\mathrm{Sm}^{3+}$ binds to a non-bridging oxygen at the scissile phosphate. 
Lanthanide sensing. The Dy10a DNAzyme is specific towards lanthanide ions as a group, and in particular, it is the most active with the five $\mathrm{Ln}^{3+}$ in the middle of the series. This trend is different from any known DNAzyme. We already reported four other lanthanide-specific DNAzymes, ${ }^{28-31}$ and this is the fifth one. By accumulating sufficient number of such DNAzymes, we may be able to distinct each lanthanide by designing a sensor array, mimicking the human tongue. ${ }^{52}$ To achieve this goal, we need to characterize the analytical performance of each DNAzyme. For this purpose, we designed a catalytic beacon sensor. The $3^{\prime}$-end of the substrate was labeled with a FAM fluorophore and the 5'-end of the enzyme labeled with a dark quencher (Figure 6A and Figure S5). In the initial hybridized state, the beacon has low fluorescence since the fluorophore is close to the quencher. Upon cleavage and release of the cleaved fragment, increased fluorescence is expected. ${ }^{53}$

A

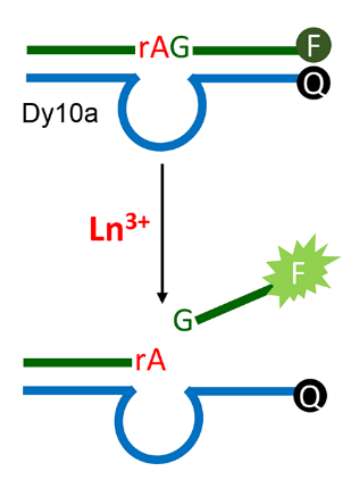

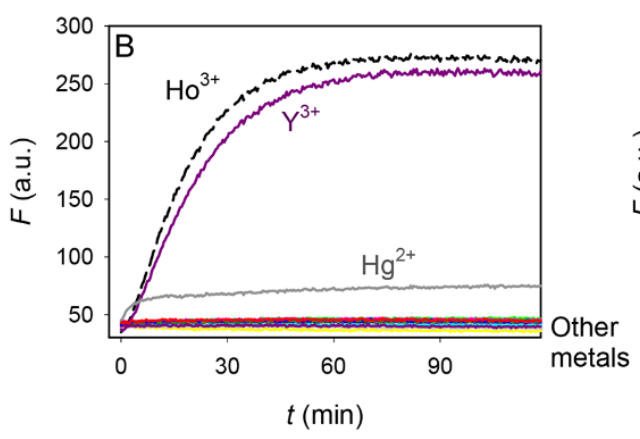

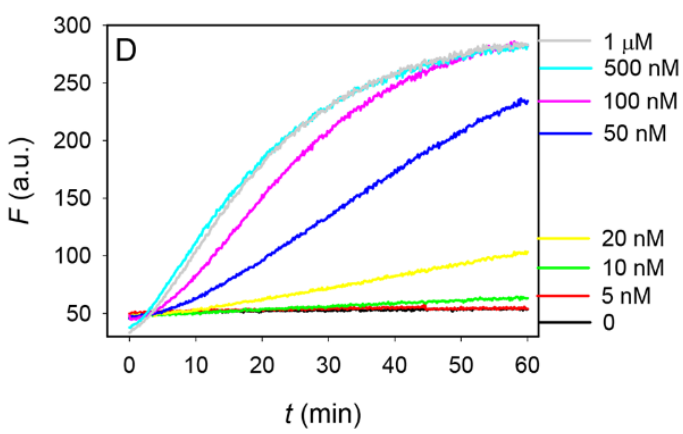

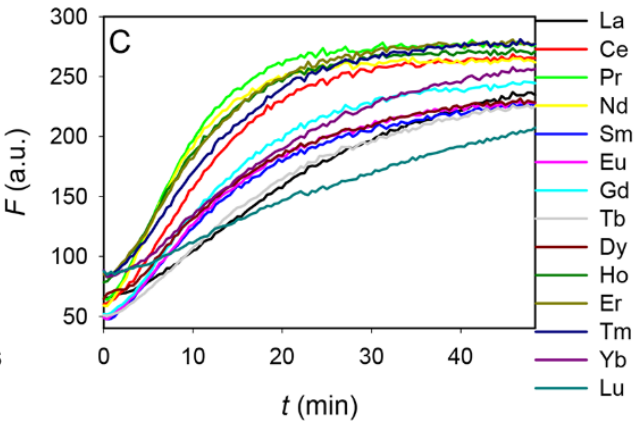

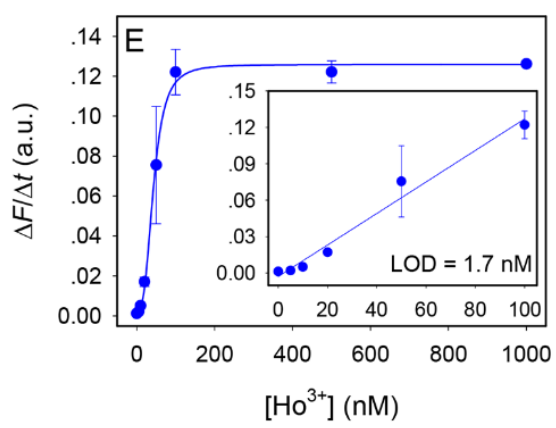

Figure 6. (A) A scheme of the Dy10a-based sensor. After cleavage and releasing of the cleaved fragment, fluorescence enhancement is observed. $\mathrm{F}=$ fluorophore; $\mathrm{Q}=$ dark quencher. (B) The 
Dy10a-based sensor ( $50 \mathrm{nM}$ ) response to $0.5 \mu \mathrm{M}$ of various metal ions. The list of the metals is in Figure 3. Sensor response to (C) $0.5 \mu \mathrm{M}$ of various $\mathrm{Ln}^{3+}$, and to (D) various concentrations of $\mathrm{Ho}^{3+}$. (E) Sensor calibration curve based on the initial rate of sensor fluorescence increase. Inset: the initial linear response at low concentrations of $\mathrm{Ho}^{3+}$.

In this experiment, we chose $\mathrm{Ho}^{3+}$ as a target, which has the highest magnetic strength among all known elements. Increased fluorescence was observed in the presence of $\mathrm{Ho}^{3+}$ and $\mathrm{Y}^{3+}$, which can cleave the substrate (Figure 6B). While most other metal ions were silent as expected, we also observed a moderate signal increase in the presence of $\mathrm{Hg}^{2+}$. A careful activity assay indicated no cleavage over a wide concentration range of $\mathrm{Hg}^{2+}$ (see Figure S6). Therefore, the $\mathrm{Hg}^{2+}$ signal might be due to other reasons such as DNAzyme folding induced by $\mathrm{Hg}^{2+}$. For example, the enzyme strand contains quite a few thymines, which may form base pairs mediated by $\mathrm{Hg}^{2+} .{ }^{44}$ Similar artifacts were observed also for an $\mathrm{Ag}^{+}$-specific DNAzyme. ${ }^{20}$ Overall, this sensor has excellent specificity consistent with the gel-based assays. We then tested all of the $\mathrm{Ln}^{3+}$ ions (Figure 6C). While signal increase was observed in each case, $\mathrm{La}^{3+}$ and $\mathrm{Lu}^{3+}$ were among the slowest, also consistent with the gel-based assay.

$\mathrm{Ho}^{3+}$ was then chosen for measuring sensitivity (Figure 6D). Interestingly, almost no signal was observed with $5 \mathrm{nM} \mathrm{Ho}^{3+}$ and a significant enhancement was achieved with 10 and 20 $\mathrm{nM} \mathrm{Ho}^{3+}$. This observation also correlates well with the cooperative metal binding observed in the gel-based assays. Figure 6E illustrates the $\mathrm{Ho}^{3+}$-concentration dependent rate of signal increase. The detection limit is $1.7 \mathrm{nM} \mathrm{Ho}^{3+}$ based on $3 \sigma / \mathrm{slope}$, where $\sigma$ is the standard deviation of background. 


\section{Conclusions.}

In summary, using a library with 50 random nucleotides, we performed in vitro selection in the presence of $\mathrm{Dy}^{3+}$ to search for new RNA-cleaving DNAzymes. While the final library was dominated by two previously reported DNAzymes (Ce13d and Lu12), we also noticed a new sequence (represented by Dy10a) that is the most active in the presence of the middle five $\operatorname{Ln}^{3+}$ in the series. Further characterizations indicate that Dy10a binds two $\mathrm{Ln}^{3+}$ ions cooperatively with a strong thio effect; the cleavage rate is suppressed by over 2500 -fold and they cannot be rescued by thiophilic $\mathrm{Cd}^{2+}$. The $\mathrm{pH}$-rate profile has a bell shape with the rising slope of 0.37 , which is different from a slope of $\sim 1$ for most known DNAzymes. Compared to previously reported Ce13d (binding one metal with a normal PS effect), Tm7 (binding three metals with completely abolished activity with the PS substrate), the property of Dy10a fits in between (binding two metals with strongly suppressed PS activity). Thus, it is an interesting new member of the lanthanide-dependent DNAzyme family. Finally, a biosensor was designed with an ability to detect $\mathrm{Ho}^{3+}$ down to $1.7 \mathrm{nM}$.

Supporting Information. DNAzyme sequences, sequence alignment, mass spectrometry data, and further biochemical characterizations. "This material is available free of charge via the Internet at http://pubs.acs.org." 


\section{References}

1. Breaker, R. R., and Joyce, G. F. (1994) A DNA enzyme that cleaves RNA, Chem. Biol. 1, 223-229.

2. Lu, Y. (2002) New transition metal-dependent DNAzymes as efficient endonucleases and as selective metal biosensors, Chem. Eur. J. 8, 4588-4596.

3. Joyce, G. F. (2004) Directed evolution of nucleic acid enzymes, Ann. Rev. Biochem. 73, $791-836$.

4. Schlosser, K., and Li, Y. F. (2009) Biologically inspired synthetic enzymes made from DNA, Chem. Biol. 16, 311-322.

5. Silverman, S. K. (2009) Deoxyribozymes: Selection design and serendipity in the development of DNA catalysts, Acc. Chem. Res. 42, 1521-1531.

6. Ward, W. L., Plakos, K., and DeRose, V. J. (2014) Nucleic acid catalysis: Metals, nucleobases, and other cofactors, Chem. Rev. 114, 4318-4342.

7. Sigel, R. K. O., and Pyle, A. M. (2007) Alternative roles for metal ions in enzyme catalysis and the implications for ribozyme chemistry, Chem. Rev. 107, 97-113.

8. Breaker, R. R., and Joyce, G. F. (1995) A DNA enzyme with $\mathrm{Mg}^{2+}$-dependent RNA phosphoesterase activity, Chem. Biol. 2, 655-660.

9. Santoro, S. W., and Joyce, G. F. (1997) A general purpose RNA-cleaving DNA enzyme, Proc. Natl. Acad. Sci. U.S.A. 94, 4262-4266.

10. Faulhammer, D., and Famulok, M. (1996) The $\mathrm{Ca}^{2+}$ ion as a cofactor for a novel RNAcleaving deoxyribozyme, Angew. Chem., Int. Ed. 35, 2837-2841.

11. Cruz, R. P. G., Withers, J. B., and Li, Y. (2004) Dinucleotide junction cleavage versatility of 8-17 deoxyribozyme, Chem. Biol. 11, 57-67. 
12. Li, J., Zheng, W., Kwon, A. H., and Lu, Y. (2000) In vitro selection and characterization of a highly efficient Zn(II)-dependent RNA-cleaving deoxyribozyme, Nucleic Acids Res. $28,481-488$.

13. Liu, Z., Mei, S. H. J., Brennan, J. D., and Li, Y. (2003) Assemblage of signaling DNA enzymes with intriguing metal-ion specificities and ph dependences, J. Am. Chem. Soc. $125,7539-7545$.

14. Bruesehoff, P., J., Li, J., Augustine, A. J., and Lu, Y. (2002) Improving metal ion specificity during in vitro selection of catalytic DNA, Comb. Chem. High T. Scr. 5, 327335.

15. Santoro, S. W., Joyce, G. F., Sakthivel, K., Gramatikova, S., and Barbas, C. F., III. (2000) RNA cleavage by a DNA enzyme with extended chemical functionality, J. Am. Chem. Soc. 122, 2433-2439.

16. Huang, P.-J. J., and Liu, J. (2016) An ultrasensitive light-up $\mathrm{Cu}^{2+}$ biosensor using a new DNAzyme cleaving a phosphorothioate-modified substrate, Anal. Chem. 88, 3341-3347.

17. Huang, P.-J. J., and Liu, J. (2015) Rational evolution of $\mathrm{Cd}^{2+}$-specific dnazymes with phosphorothioate modified cleavage junction and $\mathrm{Cd}^{2+}$ sensing, Nucleic Acids Res. 43, 6125-6133.

18. Liu, J., Brown, A. K., Meng, X., Cropek, D. M., Istok, J. D., Watson, D. B., and Lu, Y. (2007) A catalytic beacon sensor for uranium with parts-per-trillion sensitivity and millionfold selectivity, Proc. Natl. Acad. Sci. U.S.A. 104, 2056-2061.

19. Hollenstein, M., Hipolito, C., Lam, C., Dietrich, D., and Perrin, D. M. (2008) A highly selective DNAzyme sensor for mercuric ions, Angew. Chem., Int. Ed. 47, 4346 - 4350.

20. Saran, R., and Liu, J. (2016) A silver DNAzyme, Anal. Chem. 88, 4014-4020. 
21. Komiyama, M., Takeda, N., and Shigekawa, H. (1999) Hydrolysis of DNA and RNA by lanthanide ions: Mechanistic studies leading to new applications, Chem. Commun., 14431451.

22. Sugimoto, N., and Ohmichi, T. (1996) Site-specific cleavage reaction catalyzed by leadzyme is enhanced by combined effect of lead and rare earth ions, FEBS Lett. 393, 97100.

23. Geyer, C. R., and Sen, D. (1998) Lanthanide probes for a phosphodiester-cleaving, leaddependent, DNAzyme, J.Mol.Biol. 275, 483-489.

24. Feig, A. L., Scott, W. G., and Uhlenbeck, O. C. (1998) Inhibition of the hammerhead ribozyme cleavage reaction by site-specific binding of Tb(III), Science 279, 81-84.

25. Kim, H.-K., Li, J., Nagraj, N., and Lu, Y. (2008) Probing metal binding in the $8-17$ DNAzyme by $\mathrm{Tb}^{\mathrm{III}}$ luminescence spectroscopy, Chem. Eur. J 14, 8696-8703.

26. Dokukin, V., and Silverman, S. K. (2012) Lanthanide ions as required cofactors for DNA catalysts, Chem. Sci. 3, 1707-1714.

27. Javadi-Zarnaghi, F., and Hobartner, C. (2013) Lanthanide cofactors accelerate DNAcatalyzed synthesis of branched RNA, J. Am. Chem. Soc. 135, 12839-12848.

28. Huang, P.-J. J., Lin, J., Cao, J., Vazin, M., and Liu, J. (2014) Ultrasensitive DNAzyme beacon for lanthanides and metal speciation, Anal. Chem. 86, 1816-1821.

29. Huang, P.-J. J., Vazin, M., and Liu, J. (2014) In vitro selection of a new lanthanidedependent DNAzyme for ratiometric sensing lanthanides, Anal. Chem. 86, 9993-9999.

30. Huang, P.-J. J., Vazin, M., Matuszek, Ż., and Liu, J. (2015) A new heavy lanthanidedependent DNAzyme displaying strong metal cooperativity and unrescuable phosphorothioate effect, Nucleic Acids Res. 43, 461-469. 
31. Zhou, W., Ding, J., and Liu, J. (2016) An efficient lanthanide-dependent DNAzyme cleaving 2'-5' linked RNA, ChemBioChem, DOI: 10.1002/cbic.201500690.

32. Vazin, M., Huang, P.-J. J., Matuszek, Ż., and Liu, J. (2015) Biochemical characterization of a lanthanide-dependent DNAzyme with normal and phosphorothioate-modified substrates, Biochemistry 54, 6132-6138.

33. Zhou, W., Zhang, Y., Huang, P.-J. J., Ding, J., and Liu, J. (2016) A DNAzyme requiring two different metal ions at two distinct sites, Nucleic Acids Res. 44, 354-363.

34. Torabi, S.-F., and Lu, Y. (2015) Identification of the same $\mathrm{Na}^{+}$-specific DNAzyme motif from two in vitro selections under different conditions, J. Mol. Evol. 81, 225-234.

35. Zuker, M. (2003) Mfold web server for nucleic acid folding and hybridization prediction, Nucleic Acids Res. 31, 3406-3415.

36. Brown, A. K., Li, J., Pavot, C. M. B., and Lu, Y. (2003) A lead-dependent DNAzyme with a two-step mechanism, Biochemistry 42, 7152-7161.

37. Hurst, P., Takasaki, B. K., and Chin, J. (1996) Rapid cleavage of RNA with a La(III) dimer, J. Am. Chem. Soc. 118, 9982-9983.

38. Irisawa, M., Takeda, N., and Komiyama, M. (1995) Synergetic catalysis by 2 nonlanthanide metal-ions for hydrolysis of diribonucleotides, Chem. Commun., 1221-1222.

39. Shan, S.-o., Yoshida, A., Sun, S., Piccirilli, J. A., and Herschlag, D. (1999) Three metal ions at the active site of the tetrahymena group I ribozyme, Proc. Natl. Acad. Sci. U.S.A. $96,12299-12304$.

40. Stahley, M. R., Adams, P. L., Wang, J., and Strobel, S. A. (2007) Structural metals in the group I intron: A ribozyme with a multiple metal ion core, J. Mol. Biol. 372, 89-102. 
41. Weinstein, L. B., Jones, B. C. N. M., Cosstick, R., and Cech, T. R. (1997) A second catalytic metal ion in a group I ribozyme, Nature 388, 805-808.

42. Tzokov, S. B., Murray, I. A., and Grasby, J. A. (2002) The role of magnesium ions and 2'-hydroxyl groups in the VS ribozyme-substrate interaction, J. Mol. Biol. 324, 215-226.

43. Dahm, S. C., Derrick, W. B., and Uhlenbeck, O. C. (1993) Evidence for the role of solvated metal hydroxide in the hammerhead cleavage mechanism, Biochemistry 32, 13040-13045.

44. Wilson, T. J., and Lilley, D. M. J. (2011) Do the hairpin and VS ribozymes share a common catalytic mechanism based on general acid-base catalysis? A critical assessment of available experimental data, $R N A$ 17, 213-221.

45. Wilson, T. J., McLeod, A. C., and Lilley, D. M. J. (2007) A guanine nucleobase important for catalysis by the VS ribozyme, EMBO J. 26, 2489-2500.

46. Burgess, J. (1978) Metal ions in solution, Ellis Horwood Ltd., Chichester.

47. Matsumura, K., and Komiyama, M. (1997) Enormously fast RNA hydrolysis by lanthanide(III) ions under physiological conditions: Eminent candidates for novel tools of biotechnology, J. Biochem. 122, 387-394.

48. Wang, S., Karbstein, K., Peracchi, A., Beigelman, L., and Herschlag, D. (1999) Identification of the hammerhead ribozyme metal ion binding site responsible for rescue of the deleterious effect of a cleavage site phosphorothioate, Biochemistry 38, 1436314378.

49. Thaplyal, P., Ganguly, A., Golden, B. L., Hammes-Schiffer, S., and Bevilacqua, P. C. (2013) Thio effects and an unconventional metal ion rescue in the genomic hepatitis delta virus ribozyme, Biochemistry 52, 6499-6514. 
50. Nawrot, B., Widera, K., Sobczak, M., Wojcik, M., and Stec, W. J. (2008) Effect of R(p) and $\mathrm{S}(\mathrm{p})$ phosphorothioate substitution at the scissile site on the cleavage activity of deoxyribozyme 10-23, Curr. Org. Chem. 12, 1004-1009.

51. Thaplyal, P., Ganguly, A., Hammes-Schiffer, S., and Bevilacqua, P. C. (2015) Inverse thio effects in the hepatitis delta virus ribozyme reveal that the reaction pathway is controlled by metal ion charge density, Biochemistry 54, 2160-2175.

52. Rakow, N. A., and Suslick, K. S. (2000) A colorimetric sensor array for odor visualization, Nature 406, 710-713.

53. Li, J., and Lu, Y. (2000) A highly sensitive and selective catalytic DNA biosensor for lead ions, J. Am. Chem. Soc. 122, 10466-10467.

54. Ono, A., and Togashi, H. (2004) Molecular sensors: Highly selective oligonucleotidebased sensor for mercury(II) in aqueous solutions, Angew. Chem., Int. Ed. 43, 4300-4302. 